\title{
The Intervening Effect of Preventive Use of Antibacterials During the Perioperative Period in Orthopedic Department
}

\author{
Hui Liu ${ }^{1}$, Suishan Qiu ${ }^{1}$, Qinai $\mathrm{Zhu}^{2}$, Lianfang Xue ${ }^{1,}$ * \\ ${ }^{1}$ Department of Pharmacy, The First Affiliated Hospital of Jinan University, Guangzhou, China \\ ${ }^{2}$ Department of Laboratory Medicine, The First Affiliated Hospital of Jinan University, Guangzhou, China \\ Email address: \\ liu_ui@hotmail.com (Hui Liu), 494490817@qq.com (Suishan Qiu),270327330@qq.com (Qinai Zhu), xlf18666@163.com (Lianfang Xue) \\ ${ }^{*}$ Corresponding author
}

\section{To cite this article:}

Hui Liu, Suishan Qiu, Qinai Zhu, Lianfang Xue. The Intervening Effect of Preventive Use of Antibacterials During the Perioperative Period in Orthopedic Department. International Journal of Infectious Diseases and Therapy. Vol. 6, No. 1, 2021, pp. 41-44.

doi: 10.11648/j.ijidt.20210601.16

Received: February 1, 2021; Accepted: February 19, 2021; Published: March 4, 2021

\begin{abstract}
Objective: To assess the effect of orthopedic perioperative antibiotic prophylaxis intervention by clinical pharmacist. Methods: 1467 hospitalized patients in department of orthopedics with perioperative records in eight months in 2019 were chosen. 597 surgical cases before intervention were sampled as the control group, and 870 surgical records were sampled as intervention group. Investigation and analysis were performed in orthopedic perioperative prophylactic antibiotics. Results: Compared with the control group, the intervention group showed increased rationalities and decreased irrationalities in perioperative antibiotic prophylaxis. The unreasonable external use of teicoplanin was significantly decreased, and the postoperative prophylaxis duration was significantly shortened. There were no significant difference in the timing of perioperative antibiotic prophylaxis between two groups. Conclusions: After interventions for the preventive use of antibacterial drugs in orthopedic perioperative period, the rational use of antibacterial drugs has been significantly improved, the irrational use of drugs has been improved, and the effect of drug treatment has been improved. However, there is still a gap between the rational use of antibacterial drugs. Further strengthen supervision and management.
\end{abstract}

Keywords: Clinical Pharmacist, Intervention, Orthopedics Department, Perioperative Antibiotic Prophylaxis

\section{Introduction}

The perioperative prophylactic application of antibiotics is mainly to prevent the bacterial infection at the surgical site and reduce the infection rate of postoperative bacteria on the surgical incision, which has an obvious effect on improving the postoperative cure rate [1]. However, the irrational use of perioperative prophylactic antibiotics leads to the increase of drug-resistant bacteria and the rate of bacterial drug resistance. To better normalize the prophylactic use of antibacterial, the National Health Commission issued the "Guiding Principles for the Clinical Application of Antibacterials" (2015 Edition) [2]. It is stipulated in the principles of perioperative prophylactic application of antibiotics that whether to prophylactic application of antibiotics should be determined by comprehensive factors such as the type of incision corresponding to different operations, the possible types of contaminating bacteria, the duration of the operation and its sensitivity to antibiotics [3, 4]. Most of the perioperative preventive administration routes are intravenous infusion, and only a few are oral administration. Intravenous infusion should be administered within 0.5 to 1 hour before the operation or at the beginning of anesthesia to ensure that the local tissues of the surgical site are exposed to antibacterial [5]. The drug has reached a drug concentration sufficient to kill the bacteria contaminated during the operation [6]. Preventive medication maintenance time: The surgical prophylactic medication time for type I, II and III incisions generally does not exceed 24 hours, and if necessary, extend to 48 hours. Excessive extension of the medication time cannot further improve the preventive effect. If the preventive medication time exceeds 48 hours, Patients are at increased risk of 
infection by resistant bacteria. In our study, Clinical pharmacists have investigated the prophylactic application of antibiotics in the orthopedics department of a hospital during the perioperative period according to the guideline.

\section{Methods}

\subsection{Setting}

This study was performed in a total of 1467 orthopedics cases of the 1900-bed University Hospital, the First Affiliated Hospital of Jinan University in China, with 597 cases before intervention and 870 cases after intervention between May 2019 and December 2019.

\subsection{Retrospective Studies}

We used the electronic hospital administrative system to identify eligible patients that received prophylactic antibacterial during perioperative period of Orthopaedic surgery. Patient characteristics (gender, age), types of surgical site incision, prophylactic use of antibacterial (dosage, application, duration, combined drugs) were included in the collection of data.

\subsection{Intervention and Criterion}

According to "Guiding Principles for the Clinical Application of Antibacterials" (2015 Edition) issued by the National Health Commission in China, pharmacist participated in the medical treatment along with the physician. The criterion of prophylactic antibacterial were made as follows: the rational antibacterial during perioperative period of Orthopaedic surgery is the first and second generation cephalosporin antibacterial drugs, such as the rational antibacterial included cefuroxime, and cefazolin. Antibiotics should be used $30 \mathrm{~min}$ before operation, and duration should be no longer than $48 \mathrm{~h}$. We focused on the use of prophylactic antibacterial, especially those used inappropriately, then discussed with the physicians of the recommendations and made better choices of clinical drug use.

\subsection{Statistics}

The Pearson's $\chi 2$ test was used for comparison of differences between pre- and post- intervention. In all tests, $* \mathrm{P}$ $<0.05$ was considered statistically significant. The statistical analysis was conducted using Microsoft Office Excel 2017 and SPSS 19.0.

\section{Results}

\subsection{Patient Characteristics}

A total of 1467 cases were enrolled, including 597 cases before the intervention and 870 cases after the intervention. As shown in Table 1, the male/female ratio, average age, age $\geq 60 \mathrm{y}$, and the percentage of type I and type II incision were of no significant difference $(\mathrm{P} \geq 0.05)$.
Table 1. Patient characteristics of patients included pre- and post-intervention.

\begin{tabular}{lllll}
\hline & \multicolumn{2}{l}{ pre-intervention } & \multicolumn{2}{l}{ post-intervention } \\
\cline { 2 - 5 } & $\mathbf{n}$ & $\mathbf{( \% )}$ & $\mathbf{n}$ & $\mathbf{( \% )}$ \\
\hline Study participants & 597 & & 870 & \\
Female & 291 & 48.74 & 369 & 42.41 \\
Age (Mean) & 45.81 & & 45 & \\
$\geq 60$ & 193 & 32.33 & 305 & 36.55 \\
Type I, II incision & 562 & 94.1 & 847 & 97.4 \\
\hline
\end{tabular}

\subsection{Rational Drug Use Index}

As shown in Table 2, a total of 1034 cases were reasonable and 433 cases were unreasonable. After the intervention, improper drug selection, improper timing of medication, and long medication time were significantly improved compared with patients in the control group $(\mathrm{P}<0.05)$

Table 2. Comparison of rational drug use index.

\begin{tabular}{lll}
\hline Rational drug use index & $\begin{array}{l}\text { Pre-intervention n } \\
\text { (\%) }\end{array}$ & Post-intervention n (\%) \\
\hline Drug selection & $260(43.55)$ & $674(77.47)$ \\
Dosage & $497(83.25)$ & $840(96.55)$ \\
Timing & $348(58.29)$ & $817(93.90)$ \\
Duration of medication & $169(28.30)$ & $752(86.21)$ \\
\hline
\end{tabular}

\subsection{Comparison of Teicoplanin Usage}

The local usage rate of Teicoplanin injection after intervention was significantly different from that before intervention $(\mathrm{P}<0.01)$, as shown in Table 3 .

Table 3. Comparison of using methods of taicolanin pre- and post-intervention.

\begin{tabular}{lll}
\hline Drug Deliever & Pre-intervention n (\%) & Post-intervention n (\%) \\
\hline Local drug administration & $82(13.74)$ & $47(5.40)$ \\
intravenous administration & $515(86.26)$ & $823(94.60)$ \\
\hline
\end{tabular}

\section{Discussion}

At present, the abuse of antibacterial drugs has become a relatively common problem. Before the intervention of clinical pharmacists in our hospital, the application of antibacterial drugs had obvious problems such as unclear indications, high starting point for drug selection, long application time, and irregular usage and dosage. After clinical pharmacists participated in the orthopedics rounds, the following aspects should be addressed Intervention with perioperative preventive medication has achieved satisfactory results [7].

Preventive antimicrobial medication should ensure that when bacteria contaminate the incision, the tissue reaches an effective drug concentration to prevent bacteria from multiplying and play the role of preventive medication [8]. The action time of antibacterial drugs should include the whole process of surgery and 4 hours after surgery. The best time for prophylactic administration of antibacterial drugs is 30 minutes before the operation during induction of anesthesia [9]. Whether or not to add drugs during the operation is determined by considering the operation time and the half-life of antibacterial drugs. Before the intervention in this study, the 
antibacterial drugs used by the patients were mainly cephalosporins and third-generation fluoroquinolones; after the intervention, the antibacterial drugs used by the patients were mainly the first and second-generation cephalosporins [10]. Antibacterial drugs are best used alone, and combined drugs need to be clearly indicated. A large number of clinical practices at home and abroad have proven that medical technology, including surgical techniques, preoperative skin preparation, postoperative wound treatment, aseptic conditions in the operating room, and aseptic operations by medical staff, will all have an impact on postoperative infection. Dealing with these links is far more important than applying antibacterial drugs [11].

The use of antibacterial drugs is an important part of orthopedic surgery, and its rational use is of great significance in reducing and reducing postoperative infection rates. However, after investigation and analysis, it is found that there are still some unreasonable aspects in the use of antibacterial drugs during the perioperative period of orthopedics, which not only fail to prevent them, but may also increase some adverse reactions $[12,13]$.

The "Guiding Principles for the Clinical Application of Antibacterial Drugs (2015 Edition)" pointed out that the topical use of antibacterial drugs should be avoided as much as possible, and the varieties mainly for systemic application should be avoided as topical drugs. Teicoplanin injection is for systemic application [14]. There is no non-injection use description in the product and manual. Such use violates the principle and belongs to the category of beyond the manual. The clinical pharmacist pointed out that teicoplanin injection for non-injection use is a drug that exceeds the drug label, and the drug used beyond the drug label is not protected by the law, and there are certain risks in its use [15]. In the intervention investigation of clinical pharmacists, it was discovered that teicoplanin injection was used locally. Teicoplanin injection products appear to be used for non-injection purposes, and powdered at the surgical site to prevent infection. In addition to teicoplanin injection for external use, part of the doctor's order records that teicoplanin is used for deployment in the operating room For bone cement, it can be seen from the results in Table 3 that the local use rate of teicoplanin before and after intervention by clinical pharmacists decreased from $13.74 \%$ to $5.40 \%$, and the difference before and after intervention was statistically significant $(\mathrm{P}<0.01)$.

\section{Conclusion}

In summary, after interventions for the preventive use of antibacterial drugs in orthopedic perioperative period, the rational use of antibacterial drugs has been significantly improved, the irrational use of drugs has been improved, and the effect of drug treatment has been improved. However, there is still a gap between the rational use of antibacterial drugs. Further strengthen supervision and management.

\section{Acknowledgements}

This work was financially supported by the research project of Guangdong Provincial Bureau of traditional Chinese Medicine (No. 20201082 and No. 20191089) and Guangdong Provincial Hospital Pharmaceutical Research Fund (No. 2020A27).

\section{References}

[1] Prokuski L, Clyburn TA, Evans RP and Moucha CS. (2011). Prophylactic antibiotics in orthopaedic surgery. Instr Course Lect 60: 545-555.

[2] Zhiming R, Zhifen X and Yinna Z. (2017). The effect of interventional antimicrobial therapy on the prevention of infection and the level of inflammatory factors in orthopedics perioperative period. Journal of North Pharmacy 17: 149-150.

[3] Rodriguez-Pardo D. (2019). Antibiotic prophylaxis in orthopaedic surgery: Clinical practice guidelines or individualized prophylaxis? Enferm Infecc Microbiol Clin 37: 557-559.

[4] Di ZHANG, Cai-hong, Xiao-yu H and Xiao-ke D. (2015). Usage and analysis of antibacterial drug in orthopedics department during perioperative period. CHINA MODERN MEDICINE 22: 163-166.

[5] Schnabel MJ, Wagenlehner F and Schneidewind L. (2019). Perioperative antibiotic prophylaxis for stone therapy. Curr Opin Urol 29: 89-95.

[6] Xupei X. (2020). Analysis of Comprehensive Intervention Effect of Preventive Application of Antibiotics in Orthopedics Perioperative Period. Chinese Remedies \& Clinics 20: 628-630.

[7] Caixia L, Songmei L and Ying Z. (2012). Effect of intervention by clinical pharmacists on unreasonable medication in orthopaedics department during perioperative period. Chin J Nosocomiol 22: 4109-4111.

[8] Tan TL, Shohat N, Rondon AJ, Foltz C, Goswami K, Ryan SP, Seyler TM and Parvizi J. (2019). Perioperative Antibiotic Prophylaxis in Total Joint Arthroplasty: A Single Dose Is as Effective as Multiple Doses. J Bone Joint Surg Am 101: 429-437.

[9] Jingrui P, Xiaofei X and Hanquan L. (2019). Analysis of Effects of Clinical Pharmacists'Intervention on Prophylactic Use of Antibiotics in Removal of Internal Fixation Device for Fracture During Perioperative Period. Evaluation and analysis of drug-use in hospitals of China 3: 375-380.

[10] Kuong EE, Ng FY, Yan CH, Fang CX and Chiu PK. (2009). Antibiotic prophylaxis after total joint replacements. Hong Kong Med J 15: 458-462.

[11] Stefansdottir A, Robertsson O, W-Dahl A, Kiernan S, Gustafson P and Lidgren L. (2009). Inadequate timing of prophylactic antibiotics in orthopedic surgery. We can do better. Acta Orthop 80: 633-638.

[12] Rizvi M, Bille B, Holtom P and Schnall SB. (2008). The role of prophylactic antibiotics in elective hand surgery. J Hand Surg Am 33: 413-420.

[13] Zhiming R, Zhifen X and Yinna Z. (2020). Effect of interventional antimicrobial therapy on prevention of infection and levels of inflammatory factors during perioperative period of orthopedics. Journal of North Pharmacy 17: 149-150. 
[14] Jian C, Hui Z and Fuxin Z. (2019). Effect of perioperative antibiotics on prevention of infection and inflammatory cytokines in orthopedic patients. The Department of Oral Medicine Electronic Magazine (Electronic Edition) 6: 124.
[15] Bryson DJ, Morris DL, Shivji FS, Rollins KR, Snape S and Ollivere BJ. (2016). Antibiotic prophylaxis in orthopaedic surgery: difficult decisions in an era of evolving antibiotic resistance. Bone Joint J 98-B: 1014-1019. 